\title{
Modelling the cushioning properties of athletic tracks
}

\author{
Luca Andena ${ }^{1,3}$, Serena Aleo ${ }^{1}$, Francesco Caimmi ${ }^{1}$, Francesco Briatico-Vangosa ${ }^{1}$, Stefano \\ Mariani $^{2,3}$, Stefano Tagliabue ${ }^{1}$, Andrea Pavan $^{1}$ \\ ${ }^{1}$ Dipartimento di Chimica, Materiali e Ingegneria Chimica, Politecnico di Milano, Italy \\ ${ }^{2}$ Dipartimento di Ingegneria Civile e Ambientale, Politecnico di Milano, Italy \\ ${ }^{3} E^{4}$ Sport Lab, Politecnico di Milano, Italy \\ Corresponding author: Luca Andena, Assistant Professor, Polymer Engineering Lab, \\ Dipartimento di Chimica, Materiali e Ingegneria Chimica "G. Natta", Politecnico di Milano, Piazza \\ Leonardo da Vinci, 32 - 20133, Milano (Italy). \\ ORCID: 0000-0001-8610-1782 \\ E-mail: luca.andena@polimi.it; \\ tel +3902 2399 3289; fax +390223993280
}

\section{Keywords}

Sports surfaces; athletic tracks; numerical modelling; force reduction; geometric structure 


\section{Abstract}

In this work a three-dimensional finite element model of athletic tracks is presented. The model is based on data from quasi-static compression tests performed on small laboratory samples, to tune the constitutive parameters. The model was validated on three different athletic tracks, considering their top and bottom layers. Model predictions compared well with the results of shock absorption tests performed using a standard artificial athlete system, with relative errors of a few percent in terms of shock absorption. The model was then used to investigate the effect of the geometric structure of different tracks on their shock absorption capabilities. In particular, a reduction in size of the bottom layer cell pattern increased cushioning; the same property was shown to depend on the pattern voids depth in a non-monotonic way. A maximum in shock absorption was found for a void depth value about $40 \%$ higher than the one currently used in the analysed track patterns.

\section{Acknowledgements}

The authors wish to thank MONDO SpA for supplying the materials; $\mathrm{LP}^{3}$ lab of Politecnico di Milano and Mr. Oscar Bressan for help in performing the experimental tests. 


\section{Introduction}

Athletic tracks house many different specialities including running, jumping and throwing. As each specialty envisages different gestures by the athlete, these surfaces are designed to reach the best compromise between the different necessities of the athletes expected to exploit them, most notably including safety and performance. Nowadays rubbery synthetic tracks substituted both grass tracks and later porous mineral surfaces nearly all over the world, thanks to their better dynamic mechanical properties and lower maintenance needs. Modern athletic tracks can be divided into two main categories, depending on their manufacture: in-situ made systems and prefabricated layers. In-situ systems include cast elastomers, resin-bound rubber crumb and composite systems which are paved directly in the field and can easily adapt to the existing foundation. However, they may present some issues of inhomogeneity especially with respect to track thickness, which may negatively affect their overall behaviour. Prefabricated systems are made by rubber mixtures, containing mineral fillers and other additives, which are industrially calendered into sheets and subsequently consolidated by chemical curing. Production under more controlled conditions generally confers to these systems more consistent properties in the whole volume. Fixed geometrical structures (e.g. rectangular or hexagonal patterns) can be formed during the calendering phase, with the aim of optimizing the track mechanical response. The International Association of Athletics Federations (IAAF) recognises the shock absorption ability of an athletic track as having a pivotal role to ensure the athletes' safety and comfort. The main parameter employed to describe the impact response of a surface is the so-called force reduction $(F R)$, defined according to:

$$
F R=\left(1-\frac{F_{t}}{F_{r}}\right)
$$

where $F_{t}$ represents the impact peak force measured on a track whereas $F_{r}$ is the reference value of peak force, obtained on a pavement foundation. To obtain approval by IAAF the track must possess a $F R$ value between $35 \%$ and $50 \%$. FR measurements can be carried out according to EN 14808 [1], using an apparatus known as the Artificial Athlete Berlin. This testing device is composed of a $20-\mathrm{kg}$ mass falling from a height of $55 \mathrm{~mm}$ onto a rigid plate placed on top of a 
spring. The reaction force is then transmitted to a load cell connected to a base plate (a steel disc, $70 \mathrm{~mm}$ in diameter), which ultimately rests on the track sample to be tested.

Previous studies [3] demonstrated that the FR parameter depends on both the track thickness and its elastic mechanical properties. A link between track compliance and running performance had been established by earlier works [4]. Numerical modelling has been identified as a valuable tool to support the analysis of the sports surface behaviour [5-6]. Following this approach, recent works dealt with a simplified two-dimensional (2D), axisymmetric finite element (FE) model of running tracks [7-8], whose parameters have been determined by mechanical lab tests on track samples. Predictions of the numerical model were validated against results of impact experiments performed using a drop tower and an Artificial Athlete Berlin on a variety of tracks possessing different mechanical characteristics. The model became a tool to explore the behaviour of existing as well as virtual tracks, investigating the relationship between $F R$ and other quantities of potential biomechanical interest, such as loading rate and energy absorption [9-11].

Track materials are often made by coupling different layers [12]: typically a top finishing layer is stacked on a bottom one, often possessing (in the case of prefabricated tracks) a patterned structure. The purpose of the former is to ensure good properties in terms of friction, wear and environmental resistance. The base layer, is instead designed to confer the desired level of cushioning to the whole track. In principle, material properties for the separate layers could be determined and combined together using a $2 \mathrm{D}$ model of the whole track: if the bottom layer of a prefabricated track possesses a given geometrical structure, however, a homogenization step is required. A drawback of this approach is that the effect of material and geometry cannot be separately analysed: each combination of rubber and geometric structure is treated as a single homogenized material.

To overcome this limitation, a few simple 3D FE cases of sports surfaces have been developed $[6,10,13]$. The present work describes the validation of a $3 D$ extension of the numerical models already developed for athletic tracks in [7-8,14]. Such a model can highlight the role of the bottom 
layer structure and guide the optimization of its geometry with the aim of improving shock absorption and energy restitution characteristics of the complete track.

\section{Materials and experimental methods}

\section{Materials}

Three non-commercial, pre-fabricated tracks were supplied by MONDO SpA., Alba (Italy): they were produced from different blends of natural rubber (NR), styrene-butadiene rubber (SBR) and ethylene-propylene-diene monomer rubber (EPDM). They are named Mondotrack 1, Mondotrack 2 and Mondotrack 3 in the following. Although all three of them consist of two co-vulcanized layers (having a different formulation), they differ in terms of material composition, individual layers thickness and bottom layer structure. Samples of the individual top and bottom layers were also individually characterized after mechanical separation.

Table 1. Characteristics of the materials investigated in the present work. Data for the complete tracks (Mondotrack 1, 2 and 3) are listed together with those of the corresponding top and bottom layers

\begin{tabular}{|c|c|c|c|c|c|}
\hline & \multirow{2}{*}{ Label } & \multirow{2}{*}{$\begin{array}{l}\text { Track name } \\
\text { and } \\
\text { bottom layer } \\
\text { structure }\end{array}$} & \multirow{2}{*}{$\begin{array}{l}\text { Thickness } \\
\text { [mm] }\end{array}$} & \multicolumn{2}{|c|}{ Material density $\left[\mathrm{kg} / \mathrm{m}^{3}\right.$} \\
\hline & & & & Apparent & True \\
\hline \multirow{3}{*}{$\begin{array}{l}\text { COMPLETE } \\
\text { TRACKS }\end{array}$} & A & Mondotrack 1 & 14.0 & & \\
\hline & $\mathrm{D}$ & Mondotrack 2 & 13.4 & & \\
\hline & $\mathrm{H}$ & Mondotrack 3 & 12.9 & & \\
\hline \multirow{3}{*}{ TOP LAYER } & $B$ & Mondotrack 1 & 6.1 & \multicolumn{2}{|c|}{846} \\
\hline & $E$ & Mondotrack 2 & 4.6 & \multicolumn{2}{|c|}{985} \\
\hline & I & Mondotrack 3 & 3.5 & \multicolumn{2}{|c|}{925} \\
\hline \multirow{3}{*}{$\begin{array}{l}\text { BOTTOM } \\
\text { LAYER }\end{array}$} & C & $\begin{array}{c}\text { Mondotrack } 1 \\
\text { Hexagonal }\end{array}$ & 7.3 & 676 & 1000 \\
\hline & $\mathrm{F}$ & $\begin{array}{c}\text { Mondotrack } 2 \\
\text { Hexagonal }\end{array}$ & 8.0 & 750 & 990 \\
\hline & L & $\begin{array}{c}\text { Mondotrack } 3 \\
\text { Rectangular }\end{array}$ & 8.5 & 752 & 1140 \\
\hline
\end{tabular}



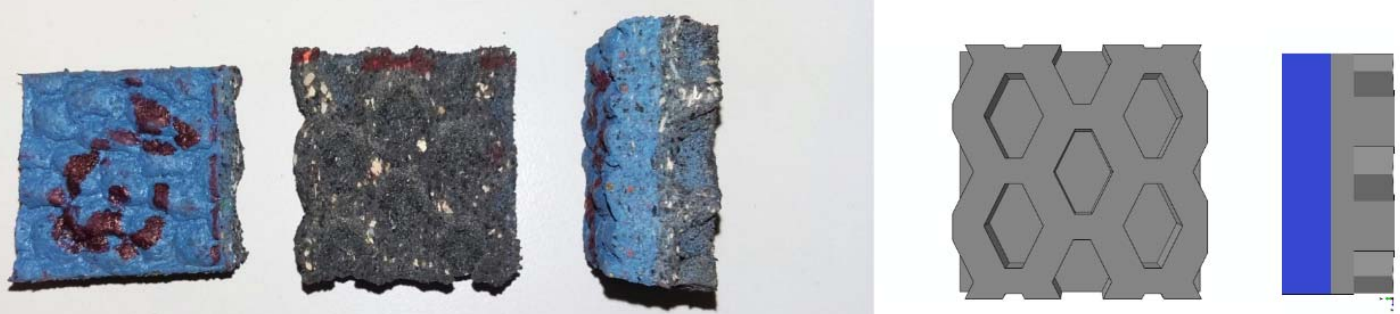

\section{Mondotrack 1}
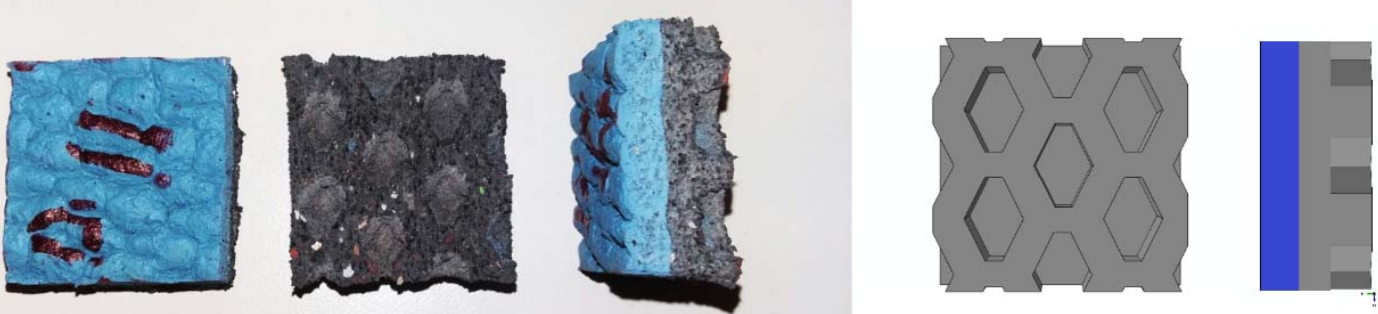

\section{Mondotrack 2}
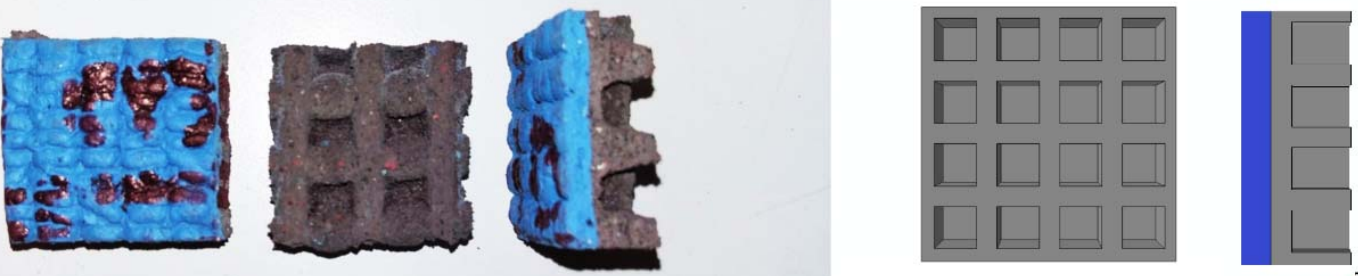

\section{Mondotrack 3}

Figure 1. On the left-hand side: photos of samples taken from the three (complete) tracks showing their overall appearance. From left to right: top view; bottom view; side view. On the right-hand side: schematics of the 3D model: bottom view (left); side view (right).

Table 1 lists the components tested, together with their labelling (as the one used in [14]), measured thickness and density. For the bottom layers, two densities are reported: an apparent density, determined as if the structure had no voids, and a true density, as measured by a pycnometer; this latter value was used for the solid material in the 3D numerical simulations. The 
total thickness of the top and bottom layers is slightly smaller than the value measured for the relevant complete tracks: the reason is that some material at the interface was removed during layer separation, to ensure planarity of the samples. Images of samples of the three complete tracks are shown in Figure 1, together with the corresponding 3D models (described later).

\section{Compression tests}

Material data within the FE simulations were obtained via quasi-static compression tests performed (along the thickness direction) on specimens cut from each complete track and from its separate top and bottom layers. The square cross section of each specimen was $30 \times 30 \mathrm{~mm}^{2}$ while the individual height was the thickness of the relevant sample as listed in Table 1. Tests were performed on a screw-driven Instron 1185R5800 electro-mechanical dynamometer under controlled environmental conditions $\left(23^{\circ} \mathrm{C}\right.$ and $\left.50 \mathrm{RH}\right)$. Three replicates were performed for each experimental condition. Each test was carried on up to a stretch ratio $\lambda=0.6 ; \lambda$ is defined as the ratio between current and initial heights of the sample, resulting in a value smaller than 1 in compression. The actual compressive deformation of the sample was determined from the crosshead displacement, corrected for the measured setup compliance. To take into account the rate-dependence of the material under test, each test was repeated for three different stretch rates $\left(-0.006,-0.06\right.$ and $\left.-0.6 \mathrm{~s}^{-1}\right)$. The testing parameters were chosen in accordance with $[11,14]$.

\section{Force Reduction tests}

$F R$ tests were performed according to IAAF standards [1-2] using an Artificial Athlete Berlin manufactured by IST (Switzerland), equipped with a Keithley analyzer. The test was repeated 3 times for each material sample (complete track). Force vs. time data from the $2^{\text {nd }}$ to $5^{\text {th }}$ tests, two more than those prescribed from the standards were performed, were processed with a low-pass second order Butterworth filter with cut-off frequency of $120 \mathrm{~Hz}$ and the resulting force peaks were averaged to obtain $F R$ values for each track/layer, as requested by IAAF standard. 


\section{Numerical simulations}

\section{Constitutive modelling}

The materials constituting the tracks' top and bottom layers were modelled using hyperelastic constitutive equations [15], as previously done in the case of 2D modelling [7-8]. They exhibit a one-to-one, reversible correspondence between stress $\sigma$ and stretch $\lambda$, in the form $\sigma=\sigma(\lambda)$, which is derived by differentiation of a strain energy function, or strain potential (per unit volume), $U$, as:

$$
U(\lambda)=\int_{0}^{\varepsilon} \sigma: d \lambda
$$

The choice of a particular strain energy function determines the hyperelastic model being used. For complete tracks and bottom layers, the incompressible version of the Mooney-Rivlin model was used, according to which the energy $U$ can be written in terms of the principal invariants $I_{1}$ and $I_{2}$ of the left Cauchy-Green strain tensor, as:

$$
U=C_{10}\left(I_{1}-3\right)+C_{01}\left(I_{2}-3\right)
$$

in which $C_{10}$ and $C_{01}$ are material (and rate) dependent parameters.

Only in the case of the separate top layers (B, E, I in Table 1) an incompressible two-term Ogden model was preferred, since it was found to give a more accurate representation of the observed mechanical behaviour in [14]. In this case, the energy $U$ can be expressed as:

$$
U=\sum_{i=1}^{2} \frac{2 \mu_{i}}{\alpha_{i}^{2}}\left(\lambda_{1}^{\alpha_{i}}+\lambda_{2}^{\alpha_{i}}+\lambda_{3}^{\alpha_{i}}-3\right)
$$

where $\lambda_{1}, \lambda_{2}$, and $\lambda_{3}$, are the principal stretches, while $\mu_{i}$ and $\alpha_{i}$ are the relevant model material parameters.

\section{Finite element modelling}

Two different 3D numerical models were considered in this study, using in both cases the generalpurpose FE code Abaqus [16].

The first model represents the actual setup used for quasi-static compression tests (see Figure 2). Material samples were explicitly modelled accounting for the geometry of the base layer (when present). The relative vertical displacement between the top and bottom surfaces of the specimen 
was imposed assuming it equal to the corrected displacement of the loading plates. Samples were meshed using eight-node hexahedral and four-node tetrahedral elements for the top and bottom layers, respectively. In both cases a hybrid formulation, particularly well-suited to treat incompressible hyperelastic materials, was used. The purpose of this model was to identify the material parameters necessary to describe the top and bottom layers, as will be explained later.

\section{1}

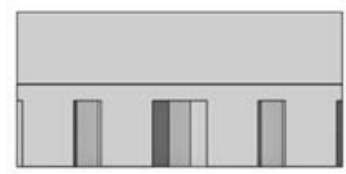

2

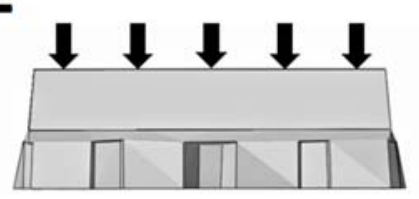

3

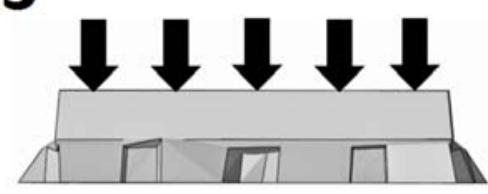

Figure 2. FE model of the uniaxial compression test: (1) sample at rest; (2) initial compression imposed by a uniform vertical displacement of the top surface; (3) sample at maximum compression, corresponding to $\lambda=0.6$.

The second model can be regarded as a 3D extension of the 2D version already described in [7], whose parts are shown in Figure 3. It closely mimics the setup of the artificial athlete testing device described in the introduction. The steel parts of the system (mass, spring, load cell and test foot) were modelled as either rigid or isotropic elastic bodies, with a Young's modulus $E_{S}=$ $200 \mathrm{GPa}$ and a Poisson's ratio $v_{s}=0.29$. The concrete foundation on which the track rests in the test was modelled as an isotropic elastic body, with Young's modulus $E_{c}=30 \mathrm{GPa}$ and Poisson's ratio $v_{c}=0.15$. The spring and the load cell were modelled as couples of thin plates connected through springs featuring an axial stiffness of $2 \mathrm{MN} / \mathrm{m}$ and 1 $\mathrm{GN} / \mathrm{m}$, and a mass of $1.46 \mathrm{~kg}$ and $0.518 \mathrm{~kg}$, respectively. In this case, the rubber material was modelled using eight-node hexahedral elements with a hybrid formulation. Only one-quarter of the testing setup was modelled thanks to the existing two-fold symmetry. In this case an explicit integration scheme was adopted to avoid convergence issues, as caused by the high number of contact interactions among rigid and highly deformable bodies. For both models, a hard contact between opposing parts (allowing no overclosure) was considered. The friction coefficient 
between steel and rubber was taken as 0.98 (as measured in [7]). The mesh density was adjusted performing a convergence study to avoid any size-dependency and to prevent spurious oscillations in the load evolution measured at the virtual load cell.

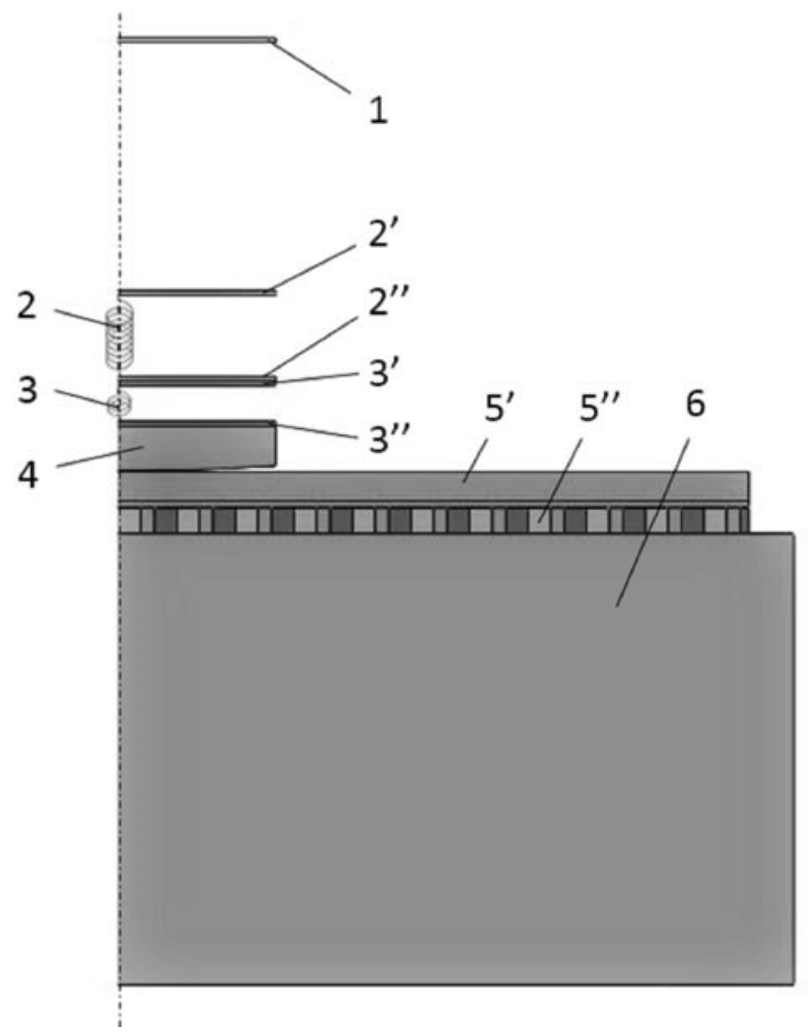

Figure 3. Schematic of the 3D Artificial Athlete Berlin numerical model (vertical cross-section): 1 Dropping mass, 2 spring (2' upper and 2" lower spring plates), 3 load cell (3' upper and 3" lower load cell plates), 4 test foot, 5 track sample (here the case of material A, composed of 5' finishing and 5" bottom layer, is shown; darker areas represent the voids of the structured bottom layer), 6 foundation.

\section{Results and discussion}

\section{Parameter identification}

Figure 4 reports typical experimental results obtained from quasi-static compression tests performed at different strain rates. As described in [11], the stress-stretch curve at the strain rate 
of $60 \mathrm{~s}^{-1}$, which is relevant to $F R$ testing, was obtained by a linear extrapolation of stress vs. log (strain rate) data.

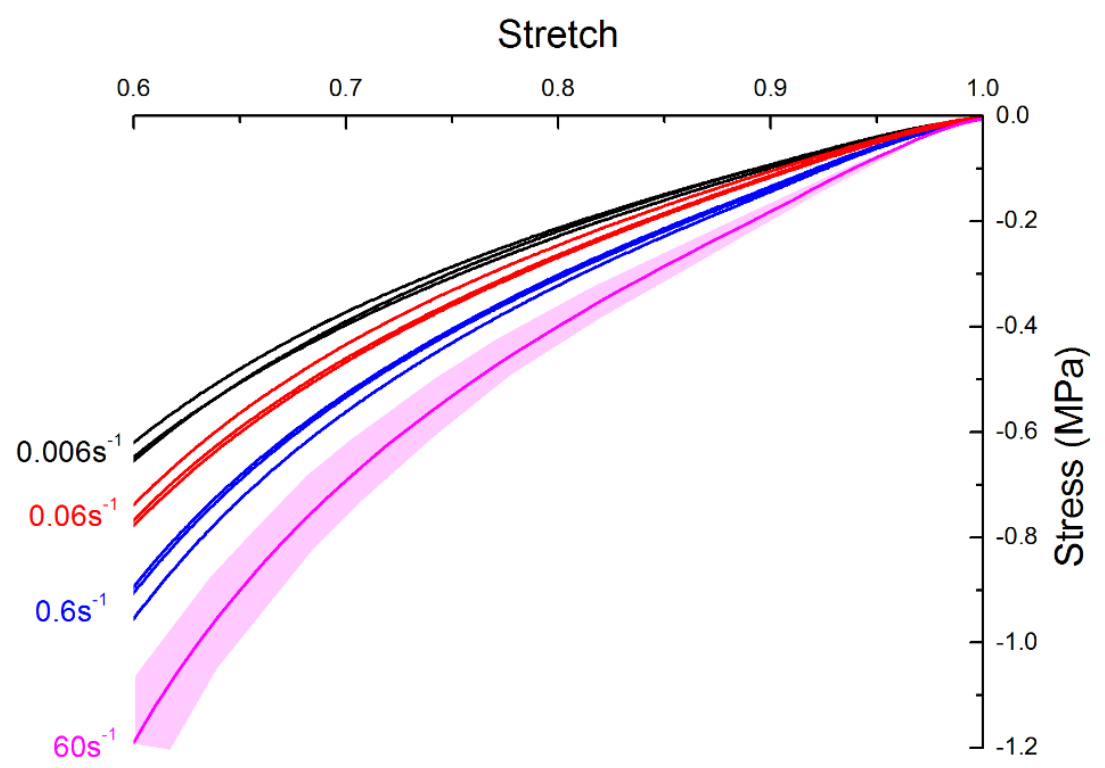

Figure 4. Nominal stress-stretch curves obtained from quasi-static compression tests performed at different strain rates $\left(0.006 \mathrm{~s}^{-1}, 0.06 \mathrm{~s}^{-1}\right.$ and $\left.0.6 \mathrm{~s}^{-1}\right)$ on track $\mathrm{A}$, and corresponding curve at 60 $\mathrm{s}^{-1}$ (the shaded area represents the $95 \%$ confidence limit associated to the extrapolation).

In a similar way, extrapolated curves at $60 \mathrm{~s}^{-1}$ were obtained for all the materials investigated: they are shown in Figure 5. The top (finishing) layers are stiffer than the bottom ones which, as expected, are closer to the corresponding complete tracks. As a whole Mondotrack 2 is stiffer than Mondotrack 1, while Mondotrack 3 displays an intermediate behaviour.
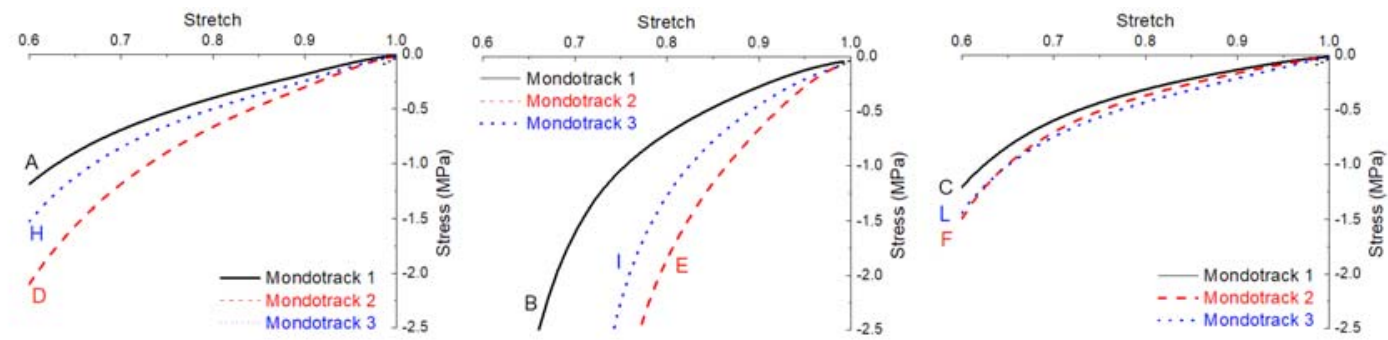
Figure 5. Extrapolated $\left(\right.$ at $\left.60 \mathrm{~s}^{-1}\right)$ stress-stretch curves for all the materials. Left: complete tracks; centre: top layers; right: bottom layers

Thanks to the substantially homogeneous nature of the top layers, compression data could be directly used to represent their inherent material behaviour in the numerical simulations. The extrapolated high-rate curves were best fitted (through least squares) to the two-term Ogden model previously described: the resulting parameters are listed in Table 2. Figure 6 shows a good agreement between extrapolated experimental data and numerical fit.

Table 2. Constitutive parameters used for modelling the materials under study.

\begin{tabular}{|c|c|c|c|c|c|c|c|}
\hline \multirow{2}{*}{ Materials } & \multirow{2}{*}{ Label } & \multicolumn{2}{|c|}{ Mooney-Rivlin Model } & \multicolumn{4}{|c|}{ Ogden Model } \\
\cline { 3 - 8 } & & $C_{10}(\mathrm{MPa})$ & $C_{01}(\mathrm{MPa})$ & $\mu_{1}(\mathrm{MPa})$ & $\alpha_{1}$ & $\mu_{2}(\mathrm{MPa})$ & $\alpha_{2}$ \\
\hline \multirow{3}{*}{ TOP LAYERS } & $\mathrm{B}$ & & & 1.03 & 8.90 & 0.00 & -18.21 \\
& $\mathrm{E}$ & & & 2.25 & 12.21 & 0.02 & -20.88 \\
& $\mathrm{I}$ & & & 1.76 & 10.14 & 0.01 & -21.51 \\
\hline \multirow{3}{*}{ BOTTOM LAYERS } & $\mathrm{C}$ & 0.12 & 0.16 & & & & \\
& $\mathrm{~F}$ & 0.06 & 0.22 & & & & \\
& $\mathrm{~L}$ & 0.38 & 0.07 & & & & \\
\hline
\end{tabular}

Stretch

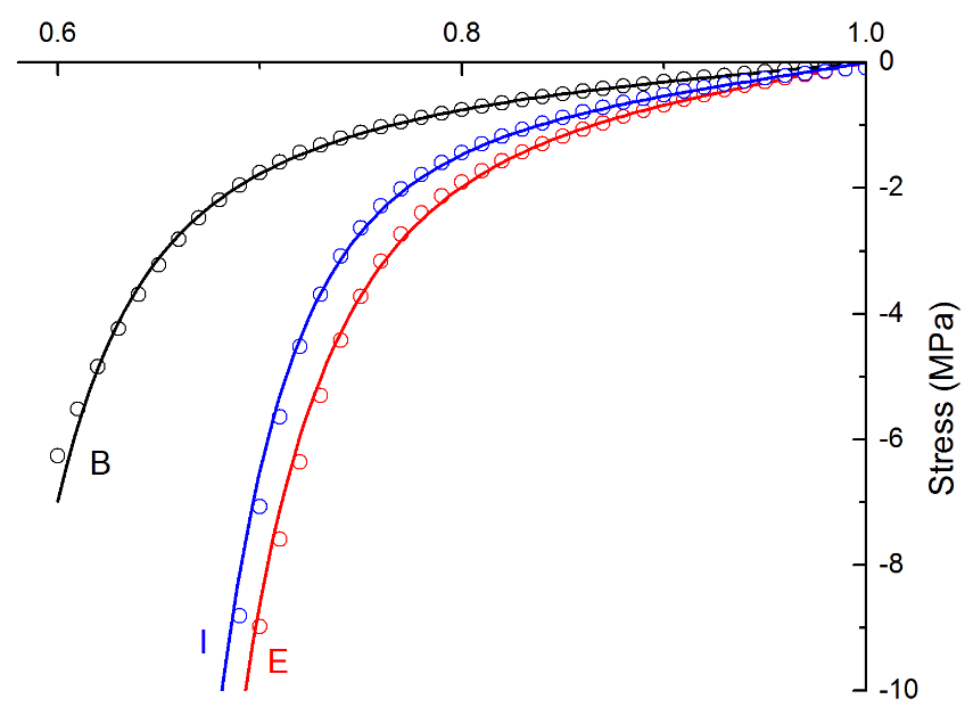

Figure 6. Experimental compression data (open circles) and numerical fit to 2-term Ogden hyperelastic model (continuous lines) for the three separate top layers 
In the case of the bottom layers, however, a direct approach is not viable. Their mechanical response results from coupling the unknown material's constitutive behaviour to the inherent geometric structure. The former cannot be directly characterized because it is not possible to obtain suitable samples from the tracks. As a consequence, an indirect identification procedure was adopted.

Simulations of the uniaxial compression tests of the three structured bottom layers $(C, F, L)$ were run, using material parameters determined by directly fitting to equation (3) the available experimental data as a first guess. It is clear that this set of effective properties, which consider the material as if it was homogeneous, is different from the true material properties because it takes into account the presence of voids. In particular, the overall stiffness is underestimated when those properties are attributed to the solid portion of the 3D model and voids are explicitly added. Consequently, parameters of the Mooney-Rivlin model (equation (3)) were tuned through an iterative procedure, until a good agreement was obtained, as shown in Figure 7. Separate graphs are displayed for the sake of clarity, as the three bottom layers exhibit similar behaviour, compared to that of the three top layers. Relevant fitting values of model parameters for the three bottom layers are listed in Table 2.

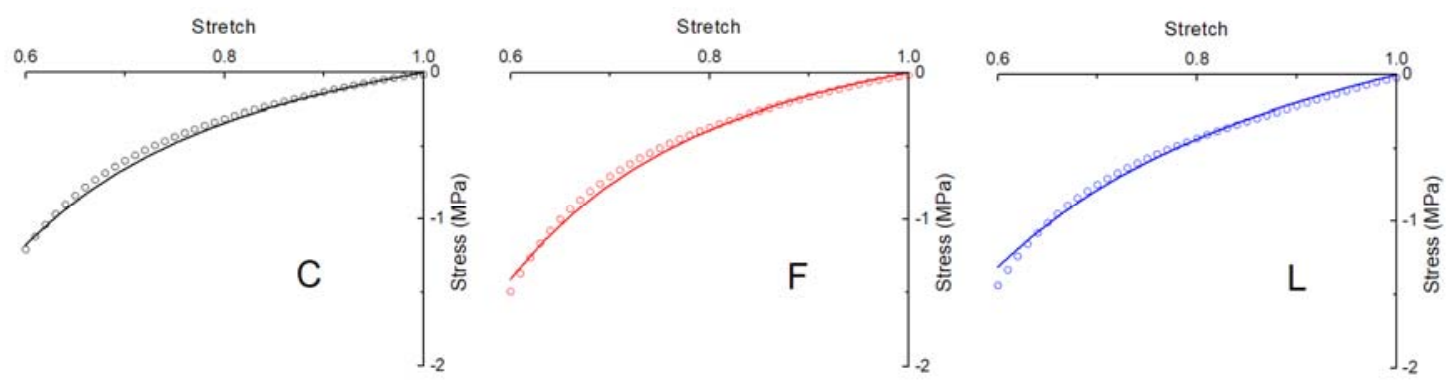

Figure 7. Experimental compression data (open circles) and numerical fit to Mooney-Rivlin hyperelastic model (continuous lines) for the three separate bottom layers

These values were used to simulate the quasi-static compression of the complete tracks combining top and bottom layers, to validate the accuracy of the identification procedure. The 
outcome of the simulations was compared with experimental data extrapolated at high strain rate $\left(60 \mathrm{~s}^{-1}\right)$ and the agreement is excellent, as shown by Figure 8 .
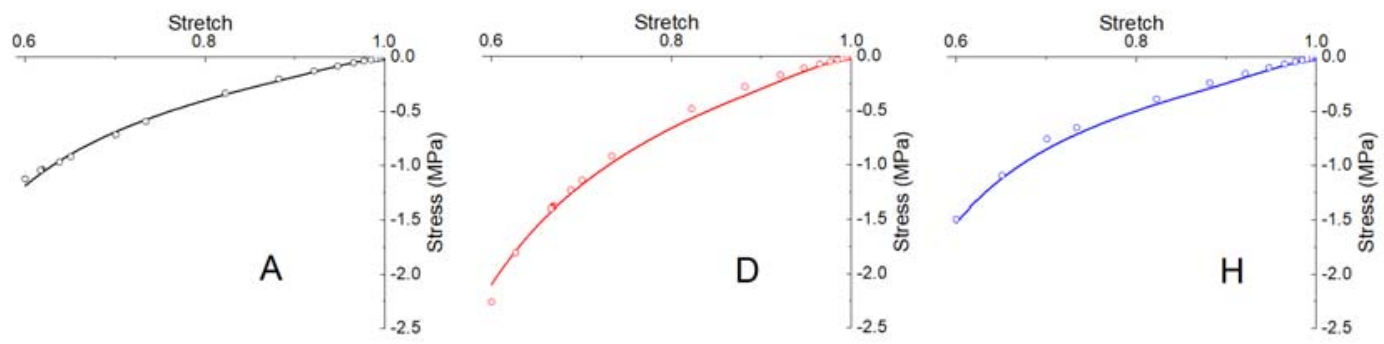

Figure 8 Extrapolated compression data (continuous lines) and results of the 3D numerical model (open circles) for the three complete tracks

\section{Force Reduction tests}

Material parameters (identified from compression tests) for the top and bottom layers were used in conjunction with the numerical 3D artificial athlete model, to simulate $F R$ tests on the complete tracks and compare the results of the simulations with experiment. The comparison is displayed in Figure 9, both in terms of raw data (top three diagrams) and filtered curves (bottom three diagrams), as explained previously in the Methods section (for the purpose of $F R$ calculation). 

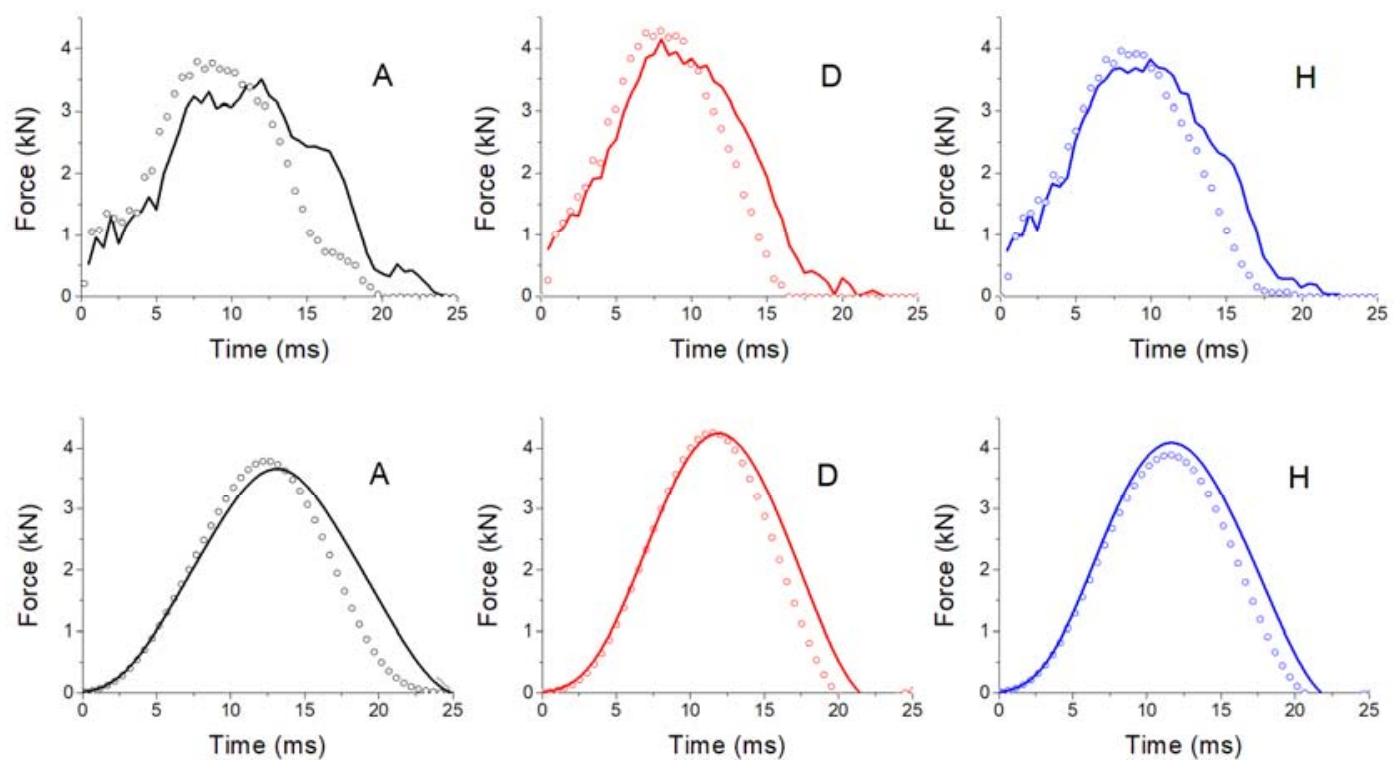

Figure 9. Experimental (open circles) and numerical (continuous lines) force vs. time curves from FR tests on the three complete tracks. Top: raw data; bottom: filtered data

In general there is a fair agreement between experimental and numerical results apart from some contact-induced oscillations present in the numerical traces. The root mean square error, RMSE - was computed on the Force vs. time curves, both filtered and unfiltered, as reported in Table 3. 
Table 3. Root mean square error (RMSE) of force vs. time curves displayed in Figure 9. Values were calculated for the whole curves and also for the loading part up to the peak value.

\begin{tabular}{c|ccccc|}
\hline \multirow{2}{*}{ Material } & \multicolumn{4}{|c|}{ RMSE [kN] } \\
\cline { 2 - 5 } & \multicolumn{2}{|c|}{ whole curve } & \multicolumn{2}{c|}{ loading curve } \\
\cline { 2 - 5 } & raw data & filtered data & raw data & filtered data \\
\hline A & 0.62 & 0.43 & 0.41 & 0.14 \\
D & 0.56 & 0.34 & 0.36 & 0.07 \\
H & 0.52 & 0.30 & 0.27 & 0.14 \\
\hline
\end{tabular}

A systematic difference between the two sets of data arises from the higher force predicted by the FE model during unloading (ref. to Figure 9). This is not surprising since the present model is hyperelastic, thus not taking into account energy dissipation within the bulk material. The highly filled rubbers used for the construction of athletic tracks have a viscoelastic behaviour, as exhibited by minor rate dependence $[3,5]$. The inherent viscoelastic relaxation is artificially factored in during the loading phase since the material parameters were determined from compression experimental tests, within a similar range of load and time. However, this pseudoelastic approach becomes inaccurate in the unloading phase. A constitutive relationship introducing material hysteresis would be required to improve the accuracy of the unloading phase, which would be important for evaluating energy return characteristics of the different tracks. However, such a task falls outside the scope of the present work. For the purpose of comparing model predictions with filtered data which was used for the calculation of $F R$, only loading data was considered (see the rightmost two columns in Table 3) and the better agreement found is consistent with calculated $F R$ values, which lie within $10 \%$ of the experimental ones, as listed in Table 4. 
Table 4. Comparison between experimental and numerical values of force reduction for the three complete tracks

\begin{tabular}{c|c|c|c}
\hline Material & $\begin{array}{c}\text { Experimental } \\
F R\end{array}$ & $\begin{array}{c}\text { Numerical } \\
F R\end{array}$ & $\begin{array}{c}\text { Relative } \\
\text { Error [\%] }\end{array}$ \\
\hline A & 39.1 & 41.2 & 5.3 \\
D & 31.5 & 31.7 & 0.5 \\
H & 37.5 & 34.2 & 8.6 \\
\hline
\end{tabular}

\section{Analysis of the effects of structured bottom layers}

Given the 3D model ability to describe the tracks' mechanical response, a parametric study on the effects caused by varying the geometric structure of the bottom layer was conducted. In particular, two parameters were considered: cell size (in-plane), and void depth (along the thickness direction); the cell shape (either parallelepipedal or prismatic with hexagonal base) and the in-plane cell pattern (either rectangular or rhombohedral grid) were kept fixed. Their meaning is explained below, with the help of Figure 10, referring to the case of a parallelepipedal cell shape and a rectangular grid.
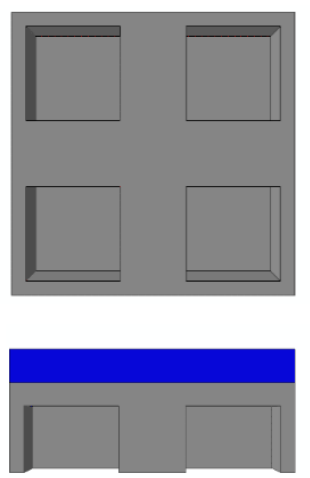
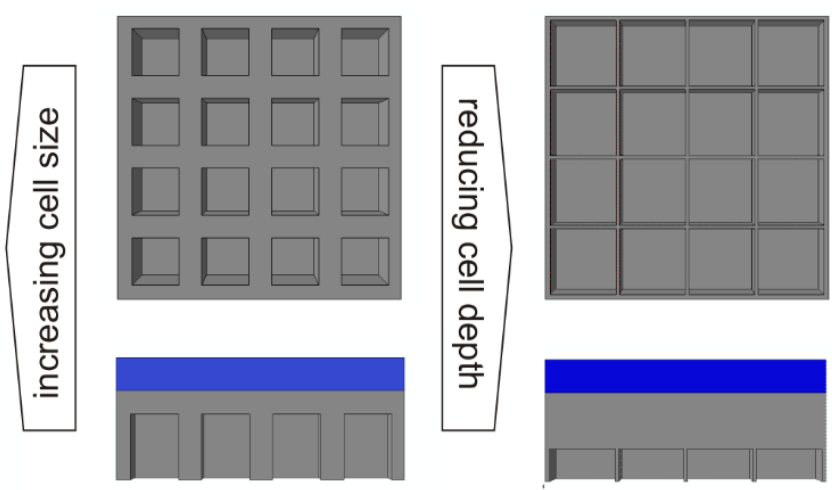

Figure 10. Bottom (above) and side view (below) examples of tracks with top (in blue) and structured bottom (in grey) layers: centre - original geometry; left - increased cell size, constant cell depth; right - reduced cell depth, constant void volumetric fraction.

Cell size was varied by scaling up or down the in-plane dimensions and the cell spacing, while cell depth was kept constant. When varying cell depth, cell spacing was instead kept constant and cell in-plane dimensions were varied to maintain the same void volumetric fraction. 
Simulations of $F R$ tests were run for the complete tracks $\mathrm{A}$ and $\mathrm{H}$ (with hexagonal prism and parallelepipedal cells, respectively). The two aforementioned geometrical parameters, cell size and void depth, were varied over the ranges $-40 \% /+80 \%$ and $-20 \% /+100 \%$, respectively, with respect to the original geometry, depicted in Figure 1. The reference values considered for the original geometry were about $10.5 \mathrm{~mm}$ for cell spacing (for both tracks) and 6 and $8 \mathrm{~mm}$ for void depth in the case of tracks $\mathrm{A}$ and $\mathrm{H}$, respectively.

The outcome of the numerical simulations is illustrated in Figure 11 for both cell size (top) and void depth (bottom).
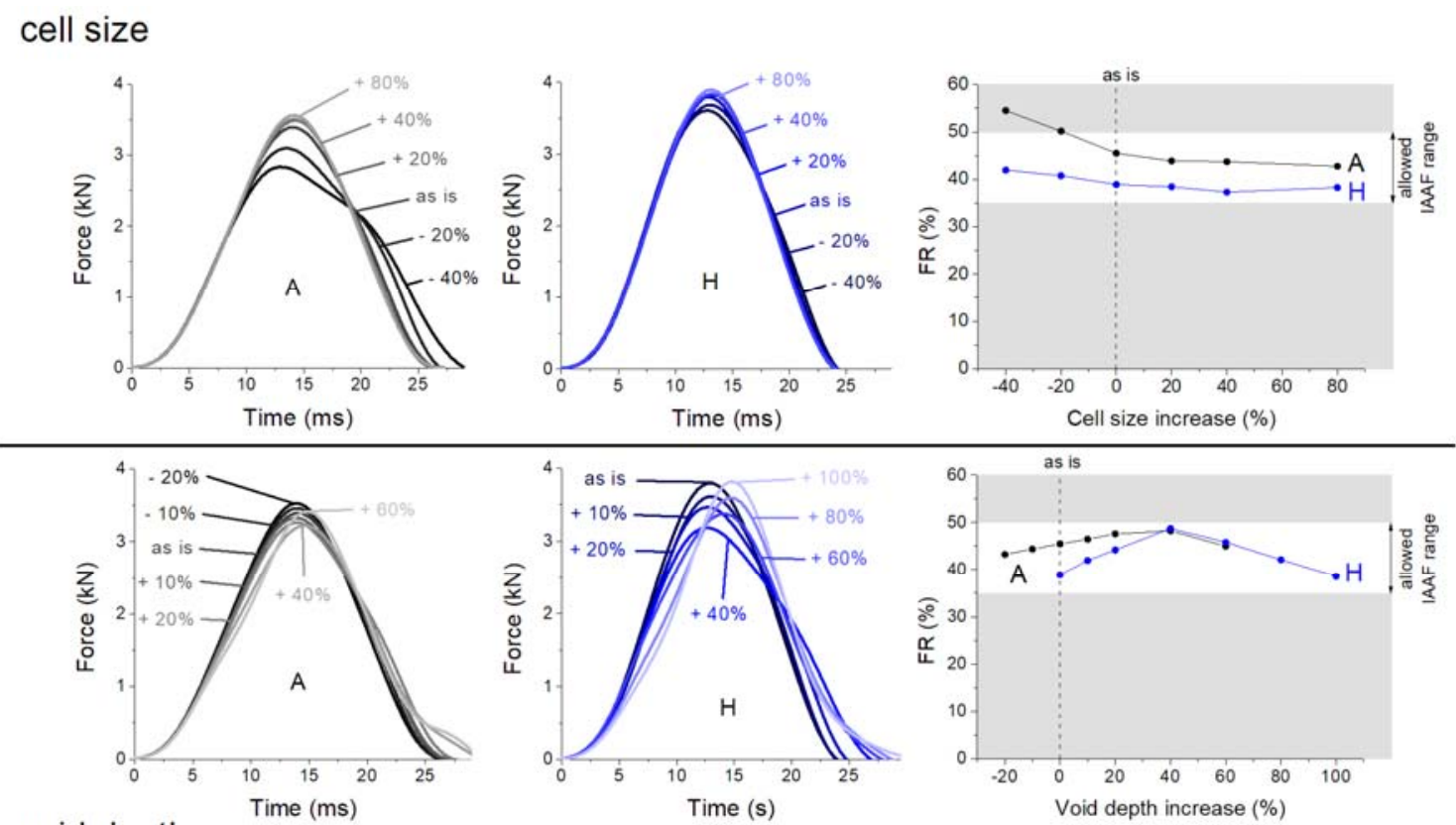

void depth

Figure 11. Effect of dimensional variations of the geometric structure of the bottom layer of tracks A and H: top - cell size; bottom - void depth. Left: force vs. time curves (simulated, filtered); right: $F R$ dependence on relevant parameter. The white band is the range of $F R$ values accepted by IAAF standards.

A reduction of the cell size of the complete track A caused a decrease of the force peak, which pushed relevant $F R$ values beyond the $50 \%$ limit set by IAAF standards. A shoulder also appeared 
in the unloading part of the force vs. time curves. Less pronounced was the effect of increasing cell size above the original value. Track $\mathrm{H}$ was less affected by a variation of cell size.

Overall, when this parameter was reduced the in-plane width of the ligaments between neighbouring cells was decreased, along with their stiffness against out-of-plane loadings. A bending-dominated deformation of the ligaments under the impact provided a decreasing peak value of the measured force, and an increase of the FR common to both types of cell patterns. An increase of the cell size did not provide a significant modification of the peak force, as the $F R$ levelled off.

An opposite behaviour was observed when considering void depth, with the larger effect displayed by track $\mathrm{H}$. In this case, the simulated force peak decreased with increasing void depth up to $+40 \%$ (with respect to the original case), and then it decreased down to the initial value (at $+100 \%$ void depth variation). Although the range variations were not the same for tracks of the A type and tracks of the $\mathrm{H}$ type (because of the different cell shape and cell pattern), a similar (but less definite) trend was found for track A. In this case, the bending deformation of the ligaments provided a major contribution to the $F R$ variation reported. Depending on the cell depth, due to the concurrent modification of ligament width and length to keep constant the void volume fraction in the structured layer, a change in the ratio between the "solid" and "hollow" volumes in the bottom layer also occurred. This fact brought about a non-monotonic variation of the peak force value and, therefore, of the $F R$.

Even though the layer is periodic only in the plane perpendicular to the impact force, some characteristics of (micro-)structured metamaterials were evidenced by the results. Primarily, the shoulder in the time histories of the force is an evidence of the dispersivity of the medium, induced by the discontinuities of the mechanical impedance witnessed by the travelling waves along their path throughout the whole track.

Changes in $F R$ values with varying geometric structure of the track bottom layer were large enough to demonstrate that there is room for optimization. The 3D model allowed a comparison of different structures, opening the way to a fine tuning of the track response. The latter could 
include reduced use of material (and related costs for production and transportation) and/or improvement of the track performance, while retaining optimal shock absorption capabilities.

\section{Discussion}

The 3D finite element model proposed in the this work is a natural extension of the previously developed 2D models presented in [7-8]. There are no additional tests required to experimentally characterize the material behaviour: the only additional step is the identification procedure applied to uniaxial compression data for the constituent material of the structured layers. The accuracy of the numerical simulations is comparable for the $2 \mathrm{D}$ and $3 \mathrm{D}$ models. Both can be used to investigate not only shock absorption, but also vertical deformation [17] , loading rate and energy absorption [11].

While the 2D model is limited to the analysis of loading states which are comparable with its axisymmetric nature, the 3D model can be used to analyse any type of loading. In particular, forces having a horizontal component can be introduced to study oblique impacts such as those occurring during actual running. The track response can thus be investigated not only in terms of its shock absorption in the vertical direction, but also of its energy return characteristics in the forward direction. At present, there is a lack of parameters able to quantify the performance of a track and this model can help with the development of new standards to certify that a given track is not only safe, but also of high performance.

The explicit introduction of the actual track geometric structure allows for its optimization, in addition to what the previous $2 \mathrm{D}$ model could do with respect to the thickness and elastic properties of the composing layers. The 3D model represents a tool capable of assisting the design of the final track product, allowing to virtually experiment with many different materials and geometric patterns without the need to produce them.

The approach adopted for the study of athletic tracks could be applied to any other piece of sports equipment made of polymeric material and undergoing quasi-static or dynamic loading including training mats, shoesand shock pads. 
The analysis herein presented is valid for materials which are solid or only slightly porous: in the case of highly porous materials (such as foams used for sports helmets, [18]) the influence of the microstructure needs to be considered as well and the material must be treated as a porous solid. Moreover, an accurate description of energy return characteristics requires addressing the material's unloading behaviour, introducing hysteresis in its constitutive law.

\section{Conclusions}

The present study detailed the development and calibration of a 3D finite element model of athletic tracks (or any other synthetic sports surface) including structured layers. The model calibration was supported by an experimental procedure which only required compression tests on small laboratory specimens at varying quasi-static testing speeds.

The ability to predict the mechanical response of athletic tracks was demonstrated by the good agreement with experimental results of $F R$ shock absorption tests performed according to IAAF standards. The model lends itself to determine additional characteristics, possibly including vertical deformation, loading rates and energy absorption. Moreover, its 3D nature makes it able to simulate impacts from different directions (i.e. not purely vertical), thus allowing the study of the interaction between the track and a running athlete, in view of improving the track performance.

The model allows the optimization of constituent materials, layer arrangement, thicknesses and geometric structure. It was found that the sensitivity of the track performance to the variation of geometrical parameters (such as cell spacing or void depth) varies for different geometric patterns. As a general trend, shock absorption was reported to increase when decreasing cell size while an increase of this geometrical parameter gave no effect within the considered range. The influence of the other considered parameter, i.e. void depth, was more complex with a nonmonotonic trend of shock absorption showing a maximum for a value which is about $40 \%$ higher than the one adopted in current pattern designs. These results show the potential for the geometry optimization which could be also extended to the response in the horizontal directions (forward and lateral). 


\section{References}

1. EN 14808:2005. Surfaces for sports areas - Determination of shock absorption.

2. IAAF 2009. Track Facilities Testing Protocols.

3. Benanti M, Andena L, Briatico-Vangosa $\mathrm{F}$, et al. Characterization of the viscoelastic behavior of athletics track surfaces in relation to their "force reduction" property. Polym Test 2013; 32:52-59.

4. MacMahon TA, Greene PR The influence of track compliance on running. J Biomech $1979 ; 12: 893-904$.

5. Carré MJ, James DM, Haake SJ. A hybrid method for assessing the performance of sports surfaces during ball impacts. Proc. Inst. Mech. Eng. L J. Mater. Des. Appl. 2006; 220:31-39.

6. Cole D, Forrester S, Fleming P. Mechanical Characterisation and Modelling of Elastomeric Shockpads. Appl. Sci. 2018; 8:501:513.

7. Andena L, Briatico-Vangosa F, Ciancio A, et al. Modeling of shock absorption in athletics track surfaces. Sports Eng 2015; 18:1-10.

8. Andena L, Briatico-Vangosa F, Cazzoni E, et al. A finite element model for the prediction of Force Reduction of athletics tracks. Proc Eng 2014; 72:847-852.

9. Nigg BM, Yeadon MR. Biomechanical aspects of playing surfaces. J Sports Sci 1987; $5: 117-145$.

10. Baroud G, Nigg BM, Stefanyshyn DJ. Energy storage and return in sport surfaces. Sports Eng 1999; 2:173-180.

11. Andena L, Ciancio A, Briatico-Vangosa F, Mariani S, Pavan A. On the relationship between force reduction, loading rate and energy absorption of athletics tracks. Proc Inst Mech Eng, Part P: J Sports Eng Tech (2018); DOI 10.1177/1754337116688577

12. IAAF. Track and Field Facilities Manual, 2008. 
13. Thomson RD, Birkbeck AE, Lucas TD. Hyperelastic modelling of nonlinear running surfaces. Sports Eng 2001, 4:215-224.

14. Andena L, Aleo S, Caimmi F, Mariani S, Briatico-Vangosa F, Pavan A. A 3D numerical model for the optimization of running tracks performance. Proc Eng 2016; 147:854-859.

15. Macosko CW. Rheology. Principles, measurements and applications. $1^{\text {st }}$ ed. New York: Wiley-VCH, 1994, p.43.

16. Dassault Systèmes. Abaqus 2016 User Manual, 2015.

17. EN 14808:2005. Surfaces for sports areas - Determination of vertical deformation.

18. Andena L, Caimmi F, Leonardi L, Ghisi A, Mariani S, Braghin F. Towards safer helmets: characterisation, modelling and monitoring. Proc Eng 2016; 147:478-483. 